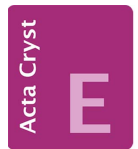
COMMUNICATIONS

ISSN 2056-9890

\section{Crystal structure of $\mathrm{Ba}_{5} \mathrm{In}_{4} \mathrm{Sb}_{6}$}

\section{Ming-Yan Pan, Sheng-Qing Xia* and Xu-Tang Tao}

State Key Laboratory of Crystal Materials, Institute of Crystal Materials, Shandong University, Jinan, Shandong 250100, People's Republic of China. *Correspondence e-mail: shqxia@sdu.edu.cn

Received 11 March 2015; accepted 7 April 2015

Edited by W. T. A. Harrison, University of Aberdeen, Scotland

The title compound, pentabarium tetraindium hexaantimony, was synthesized by an indium-flux reaction and its structure features layers composed of edge-sharing $\mathrm{In}_{2} \mathrm{Sb}_{6}$ units. The voids between the $\mathrm{In}_{4} \mathrm{Sb}_{6}$ layers are filled by $\mathrm{Ba}^{2+}$ cations, which are all surrounded by six Sb atoms and form bicapped octahedral or triangular prismatic coordination geometries. There are five barium ions in the asymmetric unit: one has no imposed crystallographic symmetry, two lie on mirror planes and two have $m m 2$ point symmetry. The two In atoms and four $\mathrm{Sb}$ atoms in the asymmetric unit all lie on general crystallographic positions.

Keywords: crystal structure; barium; indium; antimony; In—In interconnections.

CCDC reference: 1058152

\section{Related literature}

For geometrical details of In-In interconnections in other structures, see: Iandelli (1964); Goforth et al., (2008).

\section{Experimental}

2.1. Crystal data

$\mathrm{Ba}_{5} \mathrm{In}_{4} \mathrm{Sb}_{6}$

$M_{r}=1876.48$

Orthorhombic, Pmmn

$a=14.2723(13) \AA$

$b=18.3578(17) \AA$

$c=8.2710(8) \AA$

$V=2167.1(4) \AA^{3}$

$Z=4$

Mo $K \alpha$ radiation

$\mu=20.39 \mathrm{~mm}^{-1}$

$T=120 \mathrm{~K}$

$0.06 \times 0.03 \times 0.02 \mathrm{~mm}$

\subsection{Data collection}

Bruker APEXII CCD

diffractometer

Absorption correction: multi-scan

(SADABS; Bruker, 2005)

$T_{\min }=0.374, T_{\max }=0.686$

12962 measured reflections 2676 independent reflections 2098 reflections with $I>2 \sigma(I)$

$R_{\text {int }}=0.050$

\subsection{Refinement}

$R\left[F^{2}>2 \sigma\left(F^{2}\right)\right]=0.029$

$w R\left(F^{2}\right)=0.055$

$S=1.02$

2676 reflections

Data collection: APEX2 (Bruker, 2005); cell refinement: SAINT (Bruker, 2005); data reduction: $S A I N T$; $\operatorname{program}(\mathrm{s})$ used to solve structure: SHELXS97 (Sheldrick, 2008); program(s) used to refine structure: SHELXL97 (Sheldrick, 2008); molecular graphics: SHELXTL (Sheldrick, 2008); software used to prepare material for publication: SHELXTL.

\title{
Acknowledgements
}

The authors acknowledges financial support of the National Natural Science Foundation of China (grany No. 51271098).

Supporting information for this paper is available from the IUCr electronic archives (Reference: HB7382).

\section{References}

Bruker (2005). APEX2, SAINT and SADABS. Bruker AXS Inc., Madison, Wisconsin, USA.

Goforth, A. M., Klavins, P., Fettinger, J. C. \& Kauzlarich, S. M. (2008). Inorg. Chem. 47, 11048-11056.

Iandelli, A. (1964). Z. Anorg. Allg. Chem. 330, 221-232.

Sheldrick, G. M. (2008). Acta Cryst. A64, 112-122. 


\section{supporting information}

Acta Cryst. (2015). E71, i4 [https://doi.org/10.1107/S2056989015006933]

\section{Crystal structure of $\mathrm{Ba}_{5} \mathrm{In}_{4} \mathrm{Sb}_{6}$}

\section{Ming-Yan Pan, Sheng-Qing Xia and Xu-Tang Tao}

\section{S1. Comment}

Single crystal of $\mathrm{Ba}_{5} \mathrm{In}_{4} \mathrm{Sb}_{6}$ was obtained from a high-temperature In-flux reaction and it crystallizes in its own structure type. The layers are constituted by edge-shared $\mathrm{In}_{2} \mathrm{Sb}_{6}$ units and stack along the crystallographic $b$-axis direction. Each In is four-coordinated which is connected by three $\mathrm{Sb}$ and the other In atom. All Ba cations are six-coordinated with the bicapped octahedron or triangular prism geometries formed by $\mathrm{Sb}$ anions. The bond lengths of In1-In1 and In2-In2 are 2.9655 (11) and 2.8544 (11) $\AA$, respectively, comparable to In - In interconnections in $\operatorname{EuIn}_{2} \mathrm{As}_{2}$ (2.765 $\AA$ ) (Goforth et al., 2008) and $\mathrm{CaIn}_{2}(2.92 \AA)$ (Iandelli, 1964).

\section{S2. Experimental}

The title compound was synthesized through the high temperature In flux reaction. All starting elements were handled inside an Argon-filled glove box with controlled oxygen and moisture levels below 0.1 p.p.m.. The reaction conditions were optimized as follows: $\mathrm{Ba}$, In and $\mathrm{Sb}$ in a molar ratio of 3:30:5 were loaded in an alumina crucible, which were subsequently flame-sealed in a fused silica tube. The reactants were heated quickly to $1173 \mathrm{~K}$ and allowed to dwell at this temperature for $20 \mathrm{~h}$. After a slow cooling process down to $773 \mathrm{~K}$ at a rate of $2 \mathrm{~K} / \mathrm{h}$, the molten In was removed by centrifugation.

\section{S3. Refinement}

The residual electron densities show a maximum peak of $1.997 \mathrm{e} / \AA^{3}$ and a minimum hole of $-1.689 \mathrm{e} / \AA^{3}$, which are 0.78 and $1.58 \AA$ to $\mathrm{Ba} 3$ and In1, respectively. 


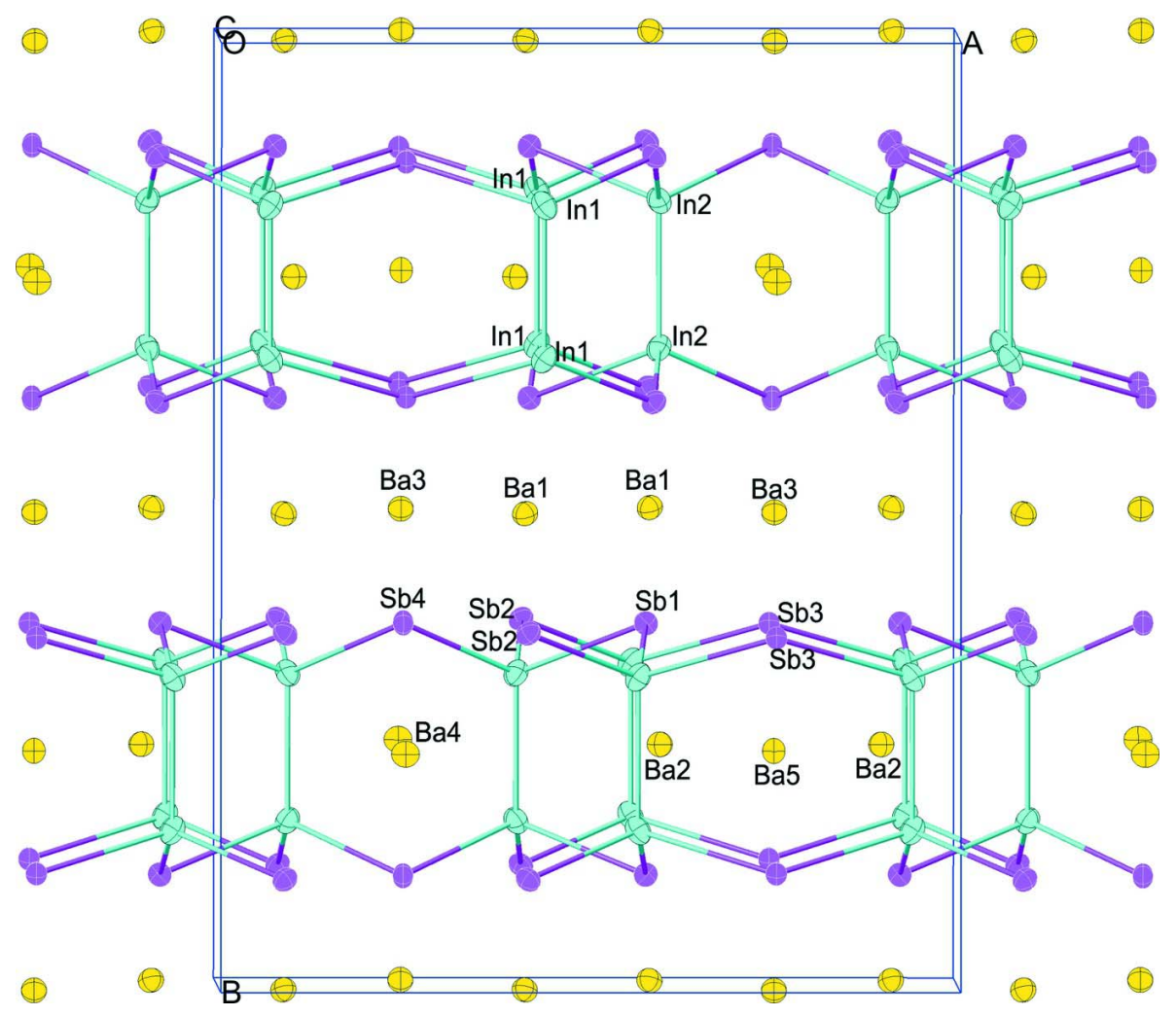

Figure 1

The ellipsoid of Ba5In4Sb6, viewed along the $c$-axis. The barium cations, Indium atoms and antimony anions were plotted as yellow, light blue and purple spheres, respectively. Ellipsoids are drawn at the $80 \%$ probability level.

Pentabarium tetraindium hexaantimony

\section{Crystal data}

$\mathrm{Ba}_{5} \mathrm{In}_{4} \mathrm{Sb}_{6}$

$M_{r}=1876.48$

Orthorhombic, $P m m n$

Hall symbol: -P 2ab 2a

$a=14.2723$ (13) $\AA$

$b=18.3578(17) \AA$

$c=8.2710(8) \AA$

$V=2167.1(4) \AA^{3}$

$Z=4$

\section{Data collection}

\section{Bruker APEXII CCD} diffractometer

Radiation source: fine-focus sealed tube Graphite monochromator $\varphi$ and $\omega$ scans

Absorption correction: multi-scan

(SADABS; Bruker, 2005)

$T_{\min }=0.374, T_{\max }=0.686$
$F(000)=3128$

$D_{\mathrm{x}}=5.751 \mathrm{Mg} \mathrm{m}^{-3}$

Mo $K \alpha$ radiation, $\lambda=0.71073 \AA$

Cell parameters from 1832 reflections

$\theta=2.9-27.6^{\circ}$

$\mu=20.39 \mathrm{~mm}^{-1}$

$T=120 \mathrm{~K}$

Block, black

$0.06 \times 0.03 \times 0.02 \mathrm{~mm}$

12962 measured reflections

2676 independent reflections

2098 reflections with $I>2 \sigma(I)$

$R_{\text {int }}=0.050$

$\theta_{\text {max }}=27.6^{\circ}, \theta_{\min }=1.8^{\circ}$

$h=-18 \rightarrow 14$

$k=-23 \rightarrow 19$

$l=-9 \rightarrow 10$ 


\section{Refinement}

Refinement on $F^{2}$

Least-squares matrix: full

$R\left[F^{2}>2 \sigma\left(F^{2}\right)\right]=0.029$

$w R\left(F^{2}\right)=0.055$

$S=1.02$

2676 reflections

79 parameters

0 restraints

Primary atom site location: structure-invariant direct methods
Secondary atom site location: difference Fourier

map

$w=1 /\left[\sigma^{2}\left(F_{\mathrm{o}}^{2}\right)+(0.0146 P)^{2}\right]$

where $P=\left(F_{\mathrm{o}}{ }^{2}+2 F_{\mathrm{c}}{ }^{2}\right) / 3$

$(\Delta / \sigma)_{\max }=0.001$

$\Delta \rho_{\max }=2.00 \mathrm{e} \AA^{-3}$

$\Delta \rho_{\min }=-1.69$ e $\AA^{-3}$

Extinction correction: SHELXL97 (Sheldrick, 2008), $\mathrm{Fc}^{*}=\mathrm{kFc}\left[1+0.001 \mathrm{xFc}^{2} \lambda^{3} / \sin (2 \theta)\right]^{-1 / 4}$

Extinction coefficient: 0.000211 (8)

Special details

Geometry. All e.s.d.'s (except the e.s.d. in the dihedral angle between two l.s. planes) are estimated using the full covariance matrix. The cell e.s.d.'s are taken into account individually in the estimation of e.s.d.'s in distances, angles and torsion angles; correlations between e.s.d.'s in cell parameters are only used when they are defined by crystal symmetry. An approximate (isotropic) treatment of cell e.s.d.'s is used for estimating e.s.d.'s involving 1.s. planes.

Refinement. Refinement of $F^{2}$ against ALL reflections. The weighted $R$-factor $w R$ and goodness of fit $S$ are based on $F^{2}$, conventional $R$-factors $R$ are based on $F$, with $F$ set to zero for negative $F^{2}$. The threshold expression of $F^{2}>\sigma\left(F^{2}\right)$ is used only for calculating $R$-factors(gt) etc. and is not relevant to the choice of reflections for refinement. $R$-factors based on $F^{2}$ are statistically about twice as large as those based on $F$, and $R$ - factors based on ALL data will be even larger.

Fractional atomic coordinates and isotropic or equivalent isotropic displacement parameters $\left(\AA^{2}\right)$

\begin{tabular}{lllll}
\hline & $x$ & $y$ & $z$ & $U_{\text {iso }} / U_{\text {eq }}$ \\
\hline Ba1 & $0.08681(3)$ & $0.00085(2)$ & $0.23996(5)$ & $0.00924(11)$ \\
Ba2 & $0.10037(4)$ & 0.2500 & $0.26369(7)$ & $0.00879(14)$ \\
Ba3 & 0.2500 & $0.50189(3)$ & $0.73898(7)$ & $0.00933(14)$ \\
Ba4 & 0.2500 & 0.7500 & $0.07159(11)$ & $0.0113(2)$ \\
Ba5 & 0.2500 & 0.2500 & $0.70687(10)$ & $0.00876(19)$ \\
In1 & $0.56473(4)$ & $0.66923(3)$ & $0.10245(6)$ & $0.00979(13)$ \\
In2 & $0.59568(3)$ & $0.17226(3)$ & $0.43328(6)$ & $0.00990(13)$ \\
Sb1 & $0.07830(3)$ & $0.11748(3)$ & $0.57615(6)$ & $0.00809(11)$ \\
Sb2 & $0.58565(3)$ & $0.12044(3)$ & $0.09457(6)$ & $0.00865(12)$ \\
Sb3 & 0.2500 & $0.12590(4)$ & $0.05576(8)$ & $0.00786(15)$ \\
Sb4 & 0.2500 & $0.61680(4)$ & $0.39782(9)$ & $0.00841(15)$
\end{tabular}

Atomic displacement parameters $\left(\AA^{2}\right)$

\begin{tabular}{lllllll}
\hline & $U^{11}$ & $U^{22}$ & $U^{33}$ & $U^{12}$ & $U^{13}$ & $U^{23}$ \\
\hline Ba1 & $0.0093(2)$ & $0.0090(2)$ & $0.0094(2)$ & $0.00006(17)$ & $-0.00107(17)$ & $0.00072(18)$ \\
Ba2 & $0.0094(3)$ & $0.0092(3)$ & $0.0078(3)$ & 0.000 & $0.0016(2)$ & 0.000 \\
Ba3 & $0.0094(3)$ & $0.0093(3)$ & $0.0093(3)$ & 0.000 & 0.000 & $0.0005(2)$ \\
Ba4 & $0.0116(5)$ & $0.0101(5)$ & $0.0122(5)$ & 0.000 & 0.000 & 0.000 \\
Ba5 & $0.0076(5)$ & $0.0095(4)$ & $0.0092(5)$ & 0.000 & 0.000 & 0.000 \\
In1 & $0.0105(3)$ & $0.0111(3)$ & $0.0077(3)$ & $-0.0025(2)$ & $-0.0016(2)$ & $0.0011(2)$ \\
In2 & $0.0088(3)$ & $0.0102(3)$ & $0.0107(3)$ & $0.0017(2)$ & $-0.0008(2)$ & $0.0008(2)$ \\
Sb1 & $0.0087(3)$ & $0.0088(3)$ & $0.0068(2)$ & $0.0004(2)$ & $-0.00068(19)$ & $-0.00060(18)$ \\
Sb2 & $0.0086(3)$ & $0.0092(3)$ & $0.0081(3)$ & $0.0012(2)$ & $-0.00116(19)$ & $-0.00030(19)$ \\
Sb3 & $0.0068(4)$ & $0.0081(4)$ & $0.0087(4)$ & 0.000 & 0.000 & $-0.0002(3)$
\end{tabular}


$\mathrm{Sb} 4$ $0.0063(4) \quad 0.0096(4)$

0.0093 (4)

0.000

0.000

$-0.0013(3)$

Geometric parameters (A, $\stackrel{\circ}{)}$

\begin{tabular}{|c|c|c|c|}
\hline $\mathrm{Ba} 1-\mathrm{Sb} 4^{\mathrm{i}}$ & $3.4343(7)$ & $\mathrm{Ba} 5-\operatorname{In} 1^{\mathrm{xviii}}$ & $3.4173(7)$ \\
\hline $\mathrm{Ba} 1-\mathrm{Sb} 2^{\mathrm{ii}}$ & $3.5105(7)$ & $\mathrm{Ba} 5-\operatorname{In} 1^{\mathrm{xix}}$ & $3.4173(7)$ \\
\hline $\mathrm{Ba} 1-\mathrm{Sb} 1$ & $3.5115(7)$ & $\mathrm{Ba} 5-\operatorname{In} 1^{\mathrm{xx}}$ & $3.4173(7)$ \\
\hline $\mathrm{Ba} 1-\mathrm{Sb} 1^{\mathrm{iii}}$ & $3.5475(7)$ & $\mathrm{Ba} 5-\mathrm{Sb} 1$ & $3.6184(6)$ \\
\hline $\mathrm{Ba} 1-\mathrm{Sb} 2^{\mathrm{iv}}$ & $3.5516(7)$ & $\mathrm{Ba} 5-\mathrm{Sb} 1^{\mathrm{i}}$ & $3.6184(6)$ \\
\hline $\mathrm{Ba} 1-\mathrm{Sb} 3$ & $3.6077(7)$ & $\mathrm{Ba} 5-\mathrm{Sb} 1^{\mathrm{ix}}$ & $3.6184(6)$ \\
\hline $\mathrm{Ba} 1-\operatorname{In} 1^{\mathrm{i}}$ & $3.9649(7)$ & $\mathrm{Ba} 5-\mathrm{Sb} 1^{\mathrm{ii}}$ & $3.6184(6)$ \\
\hline $\mathrm{Ba} 1-\mathrm{Ba} 2$ & $4.5821(6)$ & $\mathrm{Ba} 5-\mathrm{Sb} 3^{\mathrm{xii}}$ & $3.6766(10)$ \\
\hline $\mathrm{Ba} 1-\mathrm{Ba} 1^{\mathrm{ii}}$ & $4.6582(10)$ & $\mathrm{Ba} 5-\mathrm{Sb} 3^{\mathrm{xxi}}$ & $3.6766(10)$ \\
\hline $\mathrm{Ba} 1-\mathrm{Ba}^{\mathrm{v}}$ & $4.6795(9)$ & $\mathrm{Ba} 5-\mathrm{Ba} 2^{\mathrm{i}}$ & $4.2423(10)$ \\
\hline $\mathrm{Ba} 1-\mathrm{Ba} 3^{\mathrm{i}}$ & $4.7394(8)$ & In $1-\mathrm{Sb} 1^{\mathrm{xxii}}$ & $2.8296(8)$ \\
\hline $\mathrm{Ba} 1-\mathrm{Ba} 3^{\mathrm{vi}}$ & $4.7537(8)$ & $\operatorname{In} 1-\mathrm{Sb} 2^{\mathrm{xv}}$ & $2.8398(7)$ \\
\hline $\mathrm{Ba} 2-\mathrm{In} 1^{\mathrm{vii}}$ & $3.4100(8)$ & $\operatorname{In} 1-\operatorname{In} 1^{x x i i i}$ & $2.9655(11)$ \\
\hline $\mathrm{Ba} 2-\operatorname{In} 1^{\text {viii }}$ & $3.4100(8)$ & $\operatorname{In} 1-\mathrm{Sb}^{\mathrm{xxiv}}$ & $3.0556(7)$ \\
\hline $\mathrm{Ba} 2-\mathrm{In} 2^{\mathrm{ii}}$ & $3.4400(8)$ & $\operatorname{In} 1-\mathrm{Ba} 2^{\text {xxiv }}$ & $3.4100(8)$ \\
\hline $\mathrm{Ba} 2-\operatorname{In} 2^{\mathrm{i}}$ & $3.4400(8)$ & In $1-\mathrm{Ba}^{\mathrm{xvii}}$ & $3.4173(7)$ \\
\hline $\mathrm{Ba} 2-\mathrm{Sb} 1^{\mathrm{ix}}$ & $3.5632(7)$ & $\mathrm{In} 1-\mathrm{Ba} 1^{\mathrm{i}}$ & $3.9649(7)$ \\
\hline $\mathrm{Ba} 2-\mathrm{Sb} 1$ & $3.5632(7)$ & $\mathrm{In} 2-\mathrm{Sb} 4^{\mathrm{xxv}}$ & $2.7998(7)$ \\
\hline $\mathrm{Ba} 2-\mathrm{Sb}^{\mathrm{i}}$ & $3.5649(7)$ & $\operatorname{In} 2-\operatorname{In} 2^{\mathrm{ix}}$ & $2.8544(11)$ \\
\hline $\mathrm{Ba} 2-\mathrm{Sb} 3$ & $3.5649(7)$ & $\mathrm{In} 2-\mathrm{Sb} 1^{\mathrm{ii}}$ & $2.9279(7)$ \\
\hline $\mathrm{Ba} 2-\mathrm{Sb} 2^{\mathrm{ii}}$ & $3.8291(7)$ & $\mathrm{In} 2-\mathrm{Sb} 2$ & $2.9620(8)$ \\
\hline $\mathrm{Ba} 2-\mathrm{Sb} 2^{\mathrm{i}}$ & $3.8291(7)$ & $\operatorname{In} 2-\mathrm{Ba}^{\mathrm{i}}$ & $3.4400(8)$ \\
\hline $\mathrm{Ba} 2-\mathrm{Ba} 5$ & $4.2423(10)$ & $\operatorname{In} 2-\mathrm{Ba}^{\mathrm{xxv}}$ & $4.0820(8)$ \\
\hline $\mathrm{Ba} 2-\mathrm{Ba} 2^{\mathrm{i}}$ & $4.2711(13)$ & $\mathrm{Sb} 1-\operatorname{In} 1^{\mathrm{xx}}$ & $2.8296(8)$ \\
\hline $\mathrm{Ba} 3-\mathrm{Sb} 2^{\mathrm{x}}$ & $3.4833(7)$ & $\mathrm{Sb} 1-\mathrm{In} 2^{\mathrm{ii}}$ & $2.9279(7)$ \\
\hline $\mathrm{Ba} 3-\mathrm{Sb} 2^{\mathrm{xi}}$ & $3.4833(7)$ & $\mathrm{Sb} 1-\mathrm{Ba} 1^{\mathrm{iii}}$ & $3.5475(7)$ \\
\hline $\mathrm{Ba} 3-\mathrm{Sb} 3{ }^{\mathrm{xii}}$ & $3.5169(9)$ & $\mathrm{Sb} 1-\mathrm{Ba} 3^{\mathrm{i}}$ & $3.5527(7)$ \\
\hline $\mathrm{Ba} 3-\mathrm{Sb} 4$ & $3.5230(9)$ & $\mathrm{Sb} 2-\operatorname{In} 1^{\mathrm{xxvi}}$ & $2.8398(7)$ \\
\hline $\mathrm{Ba} 3-\mathrm{Sb} 1^{\mathrm{ix}}$ & $3.5527(7)$ & $\mathrm{Sb} 2-\mathrm{Ba} 3^{\mathrm{xxv}}$ & $3.4833(7)$ \\
\hline $\mathrm{Ba} 3-\mathrm{Sb}^{\mathrm{i}}$ & $3.5527(7)$ & $\mathrm{Sb} 2-\mathrm{Ba} 1^{\mathrm{ii}}$ & $3.5105(7)$ \\
\hline $\mathrm{Ba} 3-\operatorname{In} 2^{\mathrm{x}}$ & $4.0820(8)$ & $\mathrm{Sb} 2-\mathrm{Ba} 1^{\mathrm{xxvii}}$ & $3.5516(7)$ \\
\hline $\mathrm{Ba} 3-\operatorname{In} 2^{\mathrm{xi}}$ & $4.0820(8)$ & $\mathrm{Sb} 2-\mathrm{Ba} 4^{\text {xiv }}$ & $3.6122(6)$ \\
\hline $\mathrm{Ba} 3-\mathrm{Ba} 5$ & $4.6318(7)$ & $\mathrm{Sb} 2-\mathrm{Ba} 2^{\mathrm{i}}$ & $3.8291(7)$ \\
\hline $\mathrm{Ba} 3-\mathrm{Ba}^{\mathrm{ix}}$ & $4.7394(8)$ & $\mathrm{Sb} 3-\mathrm{In} 1^{\mathrm{vii}}$ & $3.0556(7)$ \\
\hline $\mathrm{Ba} 3-\mathrm{Ba}^{\mathrm{i}}$ & $4.7394(8)$ & $\mathrm{Sb} 3-\mathrm{In} 1^{\mathrm{xxvi}}$ & $3.0556(7)$ \\
\hline $\mathrm{Ba} 3-\mathrm{Ba}^{\mathrm{xii}}$ & $4.7537(8)$ & $\mathrm{Sb} 3-\mathrm{Ba}^{\mathrm{vi}}$ & $3.5169(9)$ \\
\hline $\mathrm{Ba} 4-\mathrm{Sb} 2^{\mathrm{xiii}}$ & $3.6122(6)$ & $\mathrm{Sb} 3-\mathrm{Ba} 2^{\mathrm{i}}$ & $3.5649(7)$ \\
\hline $\mathrm{Ba} 4-\mathrm{Sb} 2^{\text {xiv }}$ & $3.6122(6)$ & $\mathrm{Sb} 3-\mathrm{Ba} 1^{\mathrm{ii}}$ & $3.6077(7)$ \\
\hline $\mathrm{Ba} 4-\mathrm{Sb} 2^{\text {viii }}$ & $3.6122(6)$ & $\mathrm{Sb} 3-\mathrm{Ba} 5^{\mathrm{xxviii}}$ & $3.6766(10)$ \\
\hline $\mathrm{Ba} 4-\mathrm{Sb} 2^{\mathrm{xv}}$ & $3.6122(6)$ & $\mathrm{Sb} 4-\operatorname{In} 2^{x}$ & $2.7998(7)$ \\
\hline $\mathrm{Ba} 4-\mathrm{Sb} 4^{\mathrm{xvi}}$ & $3.6414(10)$ & $\mathrm{Sb} 4-\operatorname{In} 2^{\mathrm{xi}}$ & $2.7998(7)$ \\
\hline $\mathrm{Ba} 4-\mathrm{Sb} 4$ & $3.6414(10)$ & $\mathrm{Sb} 4-\mathrm{Ba} 1^{\mathrm{ix}}$ & $3.4343(7)$ \\
\hline $\mathrm{Ba} 5-\mathrm{In} 1^{\mathrm{xvii}}$ & $3.4173(7)$ & $\mathrm{Sb} 4-\mathrm{Ba}^{\mathrm{i}}$ & $3.4343(7)$ \\
\hline
\end{tabular}




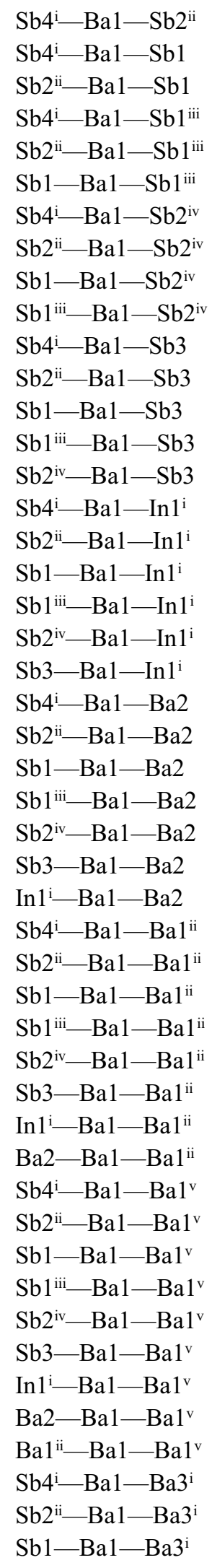

\begin{tabular}{|c|c|}
\hline $177.466(19)$ & $\mathrm{Sb} 2^{\mathrm{xiv}}-\mathrm{Ba} 4-\mathrm{Sb} 2^{\text {viii }}$ \\
\hline $96.078(18)$ & $\mathrm{Sb} 2^{\mathrm{xiii}}-\mathrm{Ba} 4-\mathrm{Sb} 2^{\mathrm{xv}}$ \\
\hline $82.282(15)$ & $\mathrm{Sb} 2^{\mathrm{xiv}}-\mathrm{Ba} 4-\mathrm{Sb} 2^{\mathrm{xv}}$ \\
\hline $84.397(16)$ & 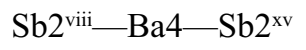 \\
\hline $93.674(16)$ & $\mathrm{Sb} 2^{\mathrm{xii}}-\mathrm{Ba} 4-\mathrm{Sb} 4^{\mathrm{xvi}}$ \\
\hline $90.625(16)$ & $\mathrm{Sb} 2^{\mathrm{xiv}}-\mathrm{Ba} 4-\mathrm{Sb} 4^{\mathrm{xvi}}$ \\
\hline $84.553(18)$ & 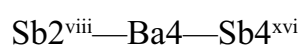 \\
\hline $97.002(15)$ & $\mathrm{Sb} 2^{\mathrm{xv}}-\mathrm{Ba} 4-\mathrm{Sb} 4^{\mathrm{xvi}}$ \\
\hline $177.436(18)$ & $\mathrm{Sb} 2^{\mathrm{xiii}}-\mathrm{Ba} 4-\mathrm{Sb} 4$ \\
\hline $86.962(15)$ & $\mathrm{Sb} 2^{\text {xiv }}-\mathrm{Ba} 4-\mathrm{Sb} 4$ \\
\hline $97.059(16)$ & $\mathrm{Sb} 2^{\text {viii_-Ba4} 4-S b 4}$ \\
\hline $84.841(15)$ & $\mathrm{Sb} 2^{\mathrm{xv}}-\mathrm{Ba} 4-\mathrm{Sb} 4$ \\
\hline $88.211(17)$ & $\mathrm{Sb} 4{ }^{\mathrm{xvi}}-\mathrm{Ba} 4-\mathrm{Sb} 4$ \\
\hline $178.223(18)$ & In $1^{\mathrm{xvii}}-\mathrm{Ba} 5-\operatorname{In} 1^{\mathrm{xviii}}$ \\
\hline $94.182(17)$ & $\operatorname{In} 1^{\mathrm{xvii}}-\mathrm{Ba} 5-\operatorname{In} 1^{\mathrm{xix}}$ \\
\hline 89.069 (17) & In $1^{\text {xviii_- }}$ Ba5-In $1^{\text {xix }}$ \\
\hline $90.672(16)$ & $\operatorname{In} 1^{\mathrm{xvii}}-\mathrm{Ba} 5-\operatorname{In} 1^{\mathrm{xx}}$ \\
\hline $133.506(16)$ & $\operatorname{In} 1^{x v i i i}-B a 5-\operatorname{In} 1^{x x}$ \\
\hline $43.815(13)$ & $\operatorname{In} 1^{x i x}-B a 5-\operatorname{In} 1^{x x}$ \\
\hline 43.967 (12) & In $1^{\text {xvii }}-\mathrm{Ba} 5-\mathrm{Sb} 1$ \\
\hline $137.062(18)$ & In $1^{\text {xviii }-B a 5-S b 1 ~}$ \\
\hline $125.651(17)$ & In $1^{\text {xix }}-\mathrm{Ba} 5-\mathrm{Sb} 1$ \\
\hline $54.554(13)$ & $\mathrm{In} 1^{\mathrm{xx}}-\mathrm{Ba} 5-\mathrm{Sb} 1$ \\
\hline $50.129(13)$ & In $1^{\text {xvii }-B a 5-S b} 1^{\mathrm{i}}$ \\
\hline $128.386(16)$ & In $1^{\text {xviii }}-\mathrm{Ba} 5-\mathrm{Sb} 1^{\mathrm{i}}$ \\
\hline $131.250(16)$ & $\mathrm{In} 1^{\mathrm{xix}}-\mathrm{Ba} 5-\mathrm{Sb} 1^{\mathrm{i}}$ \\
\hline $49.885(14)$ & $\mathrm{In} 1^{\mathrm{xx}}-\mathrm{Ba} 5-\mathrm{Sb} 1^{\mathrm{i}}$ \\
\hline $145.225(16)$ & $\mathrm{Sb} 1-\mathrm{Ba} 5-\mathrm{Sb} 1^{\mathrm{i}}$ \\
\hline $47.298(11)$ & $\mathrm{In} 1^{\mathrm{xvii}}-\mathrm{Ba} 5-\mathrm{Sb} 1^{\mathrm{ix}}$ \\
\hline $134.518(11)$ & In $1^{\text {xviii }-B a 5-S b 1^{\text {ix }}}$ \\
\hline $91.983(11)$ & $\mathrm{In} 1^{\mathrm{xix}}-\mathrm{Ba} 5-\mathrm{Sb} 1^{\mathrm{ix}}$ \\
\hline $131.624(11)$ & $\operatorname{In} 1^{\mathrm{xx}}-\mathrm{Ba} 5-\mathrm{Sb} 1^{\mathrm{ix}}$ \\
\hline $90.267(10)$ & $\mathrm{Sb} 1-\mathrm{Ba} 5-\mathrm{Sb} 1^{\mathrm{ix}}$ \\
\hline $49.790(10)$ & 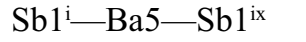 \\
\hline $123.058(10)$ & 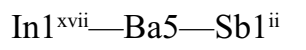 \\
\hline $87.579(9)$ & In $1^{\text {xviii }}-\mathrm{Ba} 5-\mathrm{Sb} 1^{\mathrm{ii}}$ \\
\hline $132.64(2)$ & $\mathrm{In} 1^{\mathrm{xix}}-\mathrm{Ba} 5-\mathrm{Sb} 1^{\mathrm{ii}}$ \\
\hline $48.878(11)$ & $\mathrm{In} 1^{\mathrm{xx}}-\mathrm{Ba} 5-\mathrm{Sb} 1^{\mathrm{ii}}$ \\
\hline $131.107(18)$ & $\mathrm{Sb} 1-\mathrm{Ba} 5-\mathrm{Sb} 1^{\mathrm{ii}}$ \\
\hline $90.449(16)$ & 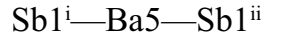 \\
\hline $48.124(12)$ & $\mathrm{Sb} 1^{\mathrm{ix}}-\mathrm{Ba} 5-\mathrm{Sb} 1^{\mathrm{ii}}$ \\
\hline $89.306(16)$ & In $1^{x v i i}-\mathrm{Ba} 5-\mathrm{Sb} 3^{\mathrm{xii}}$ \\
\hline $57.489(12)$ & In $1^{x v i i i}-\mathrm{Ba} 5-\mathrm{Sb} 3^{\mathrm{xii}}$ \\
\hline 93.748 (14) & $\mathrm{In} 1^{\mathrm{xix}}-\mathrm{Ba} 5-\mathrm{Sb} 3^{\mathrm{xii}}$ \\
\hline $121.973(11)$ & $\mathrm{In} 1^{\mathrm{xx}}-\mathrm{Ba} 5-\mathrm{Sb} 3^{\mathrm{xii}}$ \\
\hline $47.852(14)$ & $\mathrm{Sb} 1-\mathrm{Ba} 5-\mathrm{Sb} 3^{\mathrm{xii}}$ \\
\hline $130.506(15)$ & $\mathrm{Sb} 1^{\mathrm{i}}-\mathrm{Ba} 5-\mathrm{Sb} 3^{\mathrm{xii}}$ \\
\hline $48.233(12)$ & $\mathrm{Sb} 1^{\mathrm{ix}}-\mathrm{Ba} 5-\mathrm{Sb} 3^{\mathrm{xii}}$ \\
\hline
\end{tabular}

$80.989(19)$
$80.989(19)$
$82.364(19)$
$135.28(3)$
$136.392(16)$
$80.779(13)$
$80.780(13)$
$136.392(16)$
$80.780(13)$
$136.392(16)$
$136.392(16)$
$80.779(13)$
$84.37(3)$
$101.39(2)$
$51.430(19)$
$125.03(3)$
$125.03(3)$
$51.430(19)$
$101.39(2)$
$162.493(19)$
$84.581(13)$
$111.730(13)$
$47.322(13)$
$47.322(13)$
$111.730(13)$
$84.581(13)$
$162.493(19)$
$145.23(3)$
$111.730(13)$
$47.322(13)$
$162.493(19)$
$84.581(13)$
$84.494(18)$
$85.261(18)$
$84.581(13)$
$162.493(19)$
$47.322(13)$
$111.730(13)$
$85.261(18)$
$84.494(18)$
$145.23(3)$
$50.868(13)$
$50.868(13)$
$84.64(2)$
$84.64(2)$
$130.627(17)$
$79.508(13)$
$79.508(13)$

80.989 (19)

$80.989(19)$

$136.392(16)$

80.779 (13)

80.780 (13)

$136.392(16)$

80.780 (13)

$136.392(16)$

$136.392(16)$

84.37 (3)

101.39 (2)

$51.430(19)$

$125.03(3)$

$125.03(3)$

$51.430(19)$

$101.39(2)$

$162.493(19)$

84.581 (13)

$111.730(13)$

$47.322(13)$

$111.730(13)$

84.581 (13)

162.493 (19)

$145.23(3)$

$111.730(13)$

$47.322(13)$

$162.493(19)$

$84.581(13)$

$84.494(18)$

85.261 (18)

84.581 (13)

$47.322(13)$

$111.730(13)$

85.261 (18)

84.494 (18)

$145.23(3)$

50.868 (13)

$50.868(13)$

84.64 (2)

84.64 (2)

79.508 (13)

79.508 (13) 


\begin{tabular}{|c|c|}
\hline $\mathrm{Sb} 1^{\mathrm{iii}}-\mathrm{Ba} 1-\mathrm{Ba}^{\mathrm{i}}$ & $86.937(14)$ \\
\hline $\mathrm{Sb} 2^{\mathrm{iv}}-\mathrm{Ba} 1-\mathrm{Ba}^{\mathrm{i}}$ & $132.382(15)$ \\
\hline $\mathrm{Sb} 3-\mathrm{Ba} 1-\mathrm{Ba} 3^{\mathrm{i}}$ & $93.283(15)$ \\
\hline $\operatorname{In} 1^{\mathrm{i}}-\mathrm{Ba} 1-\mathrm{Ba} 3^{\mathrm{i}}$ & $120.632(14)$ \\
\hline $\mathrm{Ba} 2-\mathrm{Ba} 1-\mathrm{Ba}^{\mathrm{i}}$ & $87.281(13)$ \\
\hline $\mathrm{Ba} 1^{\mathrm{ii}}-\mathrm{Ba} 1-\mathrm{Ba} 3^{\mathrm{i}}$ & $60.565(7)$ \\
\hline $\mathrm{Ba} 1^{\mathrm{v}}-\mathrm{Ba} 1-\mathrm{Ba}^{\mathrm{i}}$ & $177.275(17)$ \\
\hline $\mathrm{Sb} 4-\mathrm{Ba} 1-\mathrm{Ba}^{\mathrm{vi}}$ & $89.567(16)$ \\
\hline $\mathrm{Sb} 2^{\mathrm{ii}}-\mathrm{Ba} 1-\mathrm{Ba}^{\mathrm{vi}}$ & $92.956(14)$ \\
\hline $\mathrm{Sb} 1-\mathrm{Ba} 1-\mathrm{Ba}^{\mathrm{vi}}$ & $135.534(16)$ \\
\hline 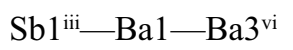 & $133.841(15)$ \\
\hline $\mathrm{Sb} 2^{\mathrm{iv}}-\mathrm{Ba} 1-\mathrm{Ba} 3^{\mathrm{vi}}$ & $46.886(12)$ \\
\hline $\mathrm{Sb} 3-\mathrm{Ba} 1-\mathrm{Ba}^{\mathrm{vi}}$ & $47.337(14)$ \\
\hline 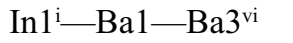 & $90.508(13)$ \\
\hline $\mathrm{Ba} 2-\mathrm{Ba} 1-\mathrm{Ba}^{\mathrm{vi}}$ & $91.559(13)$ \\
\hline $\mathrm{Ba} 1^{\mathrm{ii}}-\mathrm{Ba} 1-\mathrm{Ba} 3^{\mathrm{vi}}$ & $60.662(7)$ \\
\hline $\mathrm{Ba} 1^{\mathrm{v}}-\mathrm{Ba} 1-\mathrm{Ba}^{\mathrm{vi}}$ & $61.312(12)$ \\
\hline $\mathrm{Ba} 3^{\mathrm{i}}-\mathrm{Ba} 1-\mathrm{Ba}^{\mathrm{vi}}$ & $121.212(11)$ \\
\hline 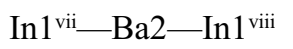 & $51.55(2)$ \\
\hline 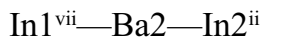 & $93.462(18)$ \\
\hline 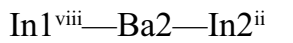 & $114.91(2)$ \\
\hline 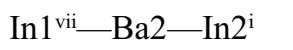 & $114.91(2)$ \\
\hline 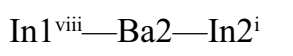 & $93.462(18)$ \\
\hline 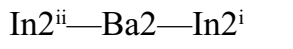 & $49.02(2)$ \\
\hline 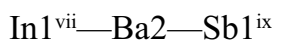 & $158.09(2)$ \\
\hline 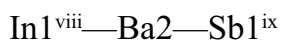 & $109.515(14)$ \\
\hline 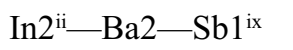 & $85.158(18)$ \\
\hline $\mathrm{In} 2^{\mathrm{i}}-\mathrm{Ba} 2-\mathrm{Sb} 1^{\mathrm{ix}}$ & $49.389(14)$ \\
\hline $\mathrm{In} 1^{\mathrm{vi}}-\mathrm{Ba} 2-\mathrm{Sb} 1$ & $109.515(14)$ \\
\hline 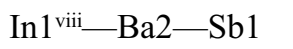 & $158.09(2)$ \\
\hline $\operatorname{In} 2^{\mathrm{ii}}-\mathrm{Ba} 2-\mathrm{Sb} 1$ & $49.389(14)$ \\
\hline $\operatorname{In} 2^{\mathrm{i}}-\mathrm{Ba} 2-\mathrm{Sb} 1$ & $85.158(18)$ \\
\hline 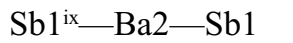 & $86.12(2)$ \\
\hline 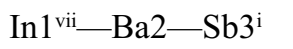 & $86.49(2)$ \\
\hline 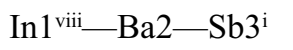 & $51.906(14)$ \\
\hline $\operatorname{In} 2^{\mathrm{ii}}-\mathrm{Ba} 2-\mathrm{Sb} 3^{\mathrm{i}}$ & $161.643(18)$ \\
\hline $\mathrm{In} 2^{\mathrm{i}}-\mathrm{Ba} 2-\mathrm{Sb} 3^{\mathrm{i}}$ & $114.761(14)$ \\
\hline $\mathrm{Sb} 1^{\mathrm{ix}}-\mathrm{Ba} 2-\mathrm{Sb}^{3}{ }^{\mathrm{i}}$ & $88.083(16)$ \\
\hline $\mathrm{Sb} 1-\mathrm{Ba} 2-\mathrm{Sb} 3^{\mathrm{i}}$ & $147.05(2)$ \\
\hline 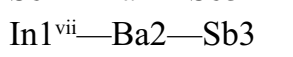 & $51.906(14)$ \\
\hline 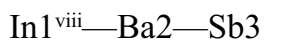 & $86.49(2)$ \\
\hline $\operatorname{In} 2^{\mathrm{ii}-}-\mathrm{Ba} 2-\mathrm{Sb} 3$ & $114.761(14)$ \\
\hline $\operatorname{In} 2-\mathrm{Ba} 2-\mathrm{Sb} 3$ & $161.643(18)$ \\
\hline $\mathrm{Sb} 1^{\mathrm{ix}}-\mathrm{Ba} 2-\mathrm{Sb} 3$ & $147.05(2)$ \\
\hline $\mathrm{Sb} 1-\mathrm{Ba} 2-\mathrm{Sb} 3$ & $88.083(16)$ \\
\hline $\mathrm{Sb} 3{ }^{\mathrm{i}}-\mathrm{Ba} 2-\mathrm{Sb} 3$ & $79.45(2)$ \\
\hline $\mathrm{In} 1^{\mathrm{vii}}-\mathrm{Ba} 2-\mathrm{Sb} 2^{\mathrm{ii}}$ & $45.739(13)$ \\
\hline In $1^{\mathrm{viii}}-\mathrm{Ba} 2-\mathrm{Sb} 2^{\mathrm{ii}}$ & $80.925(18)$ \\
\hline
\end{tabular}

\begin{tabular}{|c|c|}
\hline $\mathrm{Sb} 1^{\mathrm{ii}}-\mathrm{Ba} 5-\mathrm{Sb} 3^{\mathrm{xii}}$ & $130.627(17)$ \\
\hline In $1^{x v i i}-\mathrm{Ba} 5-\mathrm{Sb} 3^{\mathrm{xxi}}$ & $84.64(2)$ \\
\hline 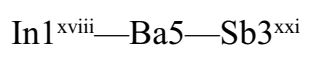 & $84.64(2)$ \\
\hline $\mathrm{In} 1^{\mathrm{xix}}-\mathrm{Ba} 5-\mathrm{Sb} 3^{\mathrm{xxi}}$ & $50.868(13)$ \\
\hline $\operatorname{In} 1^{\mathrm{xx}}-\mathrm{Ba} 5-\mathrm{Sb} 3^{\mathrm{xxi}}$ & $50.868(13)$ \\
\hline $\mathrm{Sb} 1-\mathrm{Ba} 5-\mathrm{Sb} 3^{\mathrm{xxi}}$ & $79.508(13)$ \\
\hline $\mathrm{Sb} 1^{\mathrm{i}}-\mathrm{Ba} 5-\mathrm{Sb} 3^{\mathrm{xxi}}$ & $130.627(17)$ \\
\hline $\mathrm{Sb} 1^{\mathrm{ix}}-\mathrm{Ba} 5-\mathrm{Sb} 3^{\mathrm{xxi}}$ & $130.627(17)$ \\
\hline $\mathrm{Sb} 1^{\mathrm{ii}}-\mathrm{Ba} 5-\mathrm{Sb} 3^{\mathrm{xxi}}$ & $79.508(13)$ \\
\hline $\mathrm{Sb} 3^{\mathrm{xii}}-\mathrm{Ba} 5-\mathrm{Sb} 3^{\mathrm{xxi}}$ & $76.58(3)$ \\
\hline In $1^{\text {xvii }-B a 5-B a 2}$ & $142.027(19)$ \\
\hline In $1^{\text {viii }}-\mathrm{Ba} 5-\mathrm{Ba} 2$ & $90.531(15)$ \\
\hline $\operatorname{In} 1^{\mathrm{xix}}-\mathrm{Ba} 5-\mathrm{Ba} 2$ & $142.027(19)$ \\
\hline $\operatorname{In} 1^{\mathrm{xx}}-\mathrm{Ba} 5-\mathrm{Ba} 2$ & $90.531(15)$ \\
\hline $\mathrm{Sb} 1-\mathrm{Ba} 5-\mathrm{Ba} 2$ & $53.194(13)$ \\
\hline $\mathrm{Sb} 1-\mathrm{B} a 5-\mathrm{Ba} 2$ & $94.75(2)$ \\
\hline $\mathrm{Sb} 1{ }^{\mathrm{ix}}-\mathrm{Ba} 5-\mathrm{Ba} 2$ & $53.194(13)$ \\
\hline $\mathrm{Sb} 1 \mathrm{ii}-\mathrm{Ba} 5-\mathrm{Ba} 2$ & $94.75(2)$ \\
\hline $\mathrm{Sb} 3^{\mathrm{xii}}-\mathrm{Ba} 5-\mathrm{Ba} 2$ & $132.701(10)$ \\
\hline 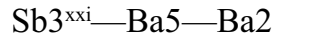 & $132.701(10)$ \\
\hline In $1^{\mathrm{xvii}}-\mathrm{Ba} 5-\mathrm{Ba} 2^{\mathrm{i}}$ & $90.531(15)$ \\
\hline In $1^{\text {xviii }}-\mathrm{Ba} 5-\mathrm{Ba} 2^{\mathrm{i}}$ & $142.027(19)$ \\
\hline $\operatorname{In} 1^{\mathrm{xix}}-\mathrm{Ba} 5-\mathrm{Ba} 2^{\mathrm{i}}$ & $90.531(15)$ \\
\hline $\mathrm{In} 1^{\mathrm{xx}}-\mathrm{Ba} 5-\mathrm{Ba} 2^{\mathrm{i}}$ & $142.027(19)$ \\
\hline $\mathrm{Sb} 1-\mathrm{Ba} 5-\mathrm{Ba}^{\mathrm{i}}$ & $94.75(2)$ \\
\hline $\mathrm{Sb} 1^{\mathrm{i}}-\mathrm{Ba} 5-\mathrm{Ba} 2^{\mathrm{i}}$ & $53.194(13)$ \\
\hline $\mathrm{Sb} 1^{\mathrm{ix}}-\mathrm{Ba} 5-\mathrm{Ba} 2^{\mathrm{i}}$ & $94.75(2)$ \\
\hline $\mathrm{Sb} 1^{\mathrm{ii}}-\mathrm{Ba} 5-\mathrm{Ba} 2^{\mathrm{i}}$ & $53.194(13)$ \\
\hline $\mathrm{Sb}^{\mathrm{xii}}-\mathrm{Ba} 5-\mathrm{Ba}^{\mathrm{i}}$ & $132.701(10)$ \\
\hline $\mathrm{Sb} 3^{\mathrm{xxi}}-\mathrm{Ba} 5-\mathrm{Ba} 2^{\mathrm{i}}$ & $132.701(10)$ \\
\hline $\mathrm{Ba} 2-\mathrm{Ba} 5-\mathrm{Ba}^{\mathrm{i}}$ & $60.45(2)$ \\
\hline $\mathrm{Sb} 1^{\mathrm{xxi}}-\mathrm{In} 1-\mathrm{Sb} 2^{\mathrm{xv}}$ & $119.01(2)$ \\
\hline 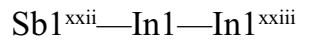 & $109.617(14)$ \\
\hline $\operatorname{Sb} 2^{\mathrm{xv}}-\operatorname{In} 1-\operatorname{In} 1^{\mathrm{xxiii}}$ & $108.387(14)$ \\
\hline $\mathrm{Sb} 1^{\mathrm{xxi}}-\mathrm{In} 1-\mathrm{Sb} 3^{\mathrm{xxiv}}$ & $104.82(2)$ \\
\hline $\mathrm{Sb} 2^{\mathrm{xv}}-\mathrm{In} 1-\mathrm{Sb} 3^{\mathrm{xxiv}}$ & $109.04(2)$ \\
\hline In $1^{\mathrm{xxii}}-\operatorname{In} 1-\mathrm{Sb} 3^{\mathrm{xxiv}}$ & $105.090(16)$ \\
\hline 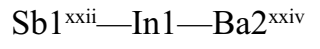 & $165.95(2)$ \\
\hline $\mathrm{Sb} 2^{\mathrm{xv}}-\mathrm{In} 1-\mathrm{Ba} 2^{\mathrm{xxiv}}$ & $74.947(19)$ \\
\hline In $1^{\text {xxiii }-I n} 1-B a 2^{\text {xxiv }}$ & $64.226(10)$ \\
\hline $\mathrm{Sb} 3^{\text {xxiv }}-\operatorname{In} 1-\mathrm{Ba} 2^{\text {xxiv }}$ & $66.660(19)$ \\
\hline $\mathrm{Sb} 1^{\mathrm{xxii}}-\operatorname{In} 1-\mathrm{Ba}^{\mathrm{xvii}}$ & $70.071(19)$ \\
\hline $\mathrm{Sb} 2^{\mathrm{xv}}-\mathrm{In} 1-\mathrm{Ba} 5^{\mathrm{xvii}}$ & $170.58(2)$ \\
\hline In $1^{\text {xxiii }}-\operatorname{In} 1-B a 5^{\text {xvii }}$ & $64.285(10)$ \\
\hline $\mathrm{Sb} 3^{\text {xiv }}-\mathrm{In} 1-\mathrm{Ba}^{\mathrm{xvii}}$ & $68.962(19)$ \\
\hline $\mathrm{Ba} 2^{\mathrm{xxiv}}-\mathrm{In} 1-\mathrm{Ba}^{\mathrm{xvii}}$ & $96.07(2)$ \\
\hline $\mathrm{Sb} 1^{\mathrm{xxii}}-\mathrm{In} 1-\mathrm{Ba} 1^{\mathrm{i}}$ & $60.226(15)$ \\
\hline $\mathrm{Sb} 2^{\mathrm{xv}}-\mathrm{In} 1-\mathrm{Ba} 1^{\mathrm{i}}$ & $60.260(15)$ \\
\hline
\end{tabular}




\begin{tabular}{|c|c|}
\hline $\operatorname{In} 2^{\mathrm{ii}}-\mathrm{Ba} 2-\mathrm{Sb} 2^{\mathrm{ii}}$ & $47.723(14)$ \\
\hline $\mathrm{In} 2^{\mathrm{i}}-\mathrm{Ba} 2-\mathrm{Sb} 2^{\mathrm{ii}}$ & $80.950(18)$ \\
\hline $\mathrm{Sb} 1^{\mathrm{ix}}-\mathrm{Ba} 2-\mathrm{Sb} 2^{\mathrm{ii}}$ & $128.88(2)$ \\
\hline $\mathrm{Sb} 1-\mathrm{Ba} 2-\mathrm{Sb} 2^{\mathrm{ii}}$ & $77.265(13)$ \\
\hline $\mathrm{Sb} 3{ }^{\mathrm{i}}-\mathrm{Ba} 2-\mathrm{Sb} 2^{\mathrm{ii}}$ & $129.50(2)$ \\
\hline $\mathrm{Sb} 3-\mathrm{Ba} 2-\mathrm{Sb} 2^{\mathrm{ii}}$ & $80.918(14)$ \\
\hline 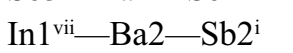 & $80.925(18)$ \\
\hline In $1^{\text {viii- }-B a 2-S b 2}{ }^{\mathrm{i}}$ & $45.739(13)$ \\
\hline $\mathrm{In} 2^{\mathrm{ii}}-\mathrm{Ba} 2-\mathrm{Sb} 2^{\mathrm{i}}$ & $80.950(18)$ \\
\hline $\operatorname{In} 2^{\mathrm{i}}-\mathrm{Ba} 2-\mathrm{Sb} 2^{\mathrm{i}}$ & $47.723(14)$ \\
\hline $\mathrm{Sb} 1^{\mathrm{ix}}-\mathrm{Ba} 2-\mathrm{Sb} 2^{\mathrm{i}}$ & $77.265(13)$ \\
\hline $\mathrm{Sb} 1-\mathrm{Ba} 2-\mathrm{Sb}^{2}$ & $128.88(2)$ \\
\hline $\mathrm{Sb} 3^{\mathrm{i}}-\mathrm{Ba} 2-\mathrm{Sb} 2^{\mathrm{i}}$ & $80.918(14)$ \\
\hline $\mathrm{Sb} 3-\mathrm{Ba} 2-\mathrm{Sb} 2^{\mathrm{i}}$ & $129.50(2)$ \\
\hline $\mathrm{Sb} 2^{\mathrm{ii}}-\mathrm{Ba} 2-\mathrm{Sb} 2^{\mathrm{i}}$ & $76.80(2)$ \\
\hline In $1^{\text {vii- }-B a 2-B a 5}$ & $147.398(15)$ \\
\hline In $1^{\text {viii_-Ba2-Ba5 }}$ & $147.398(15)$ \\
\hline In $2^{\mathrm{ii}-\mathrm{Ba} 2-\mathrm{Ba} 5}$ & $93.275(18)$ \\
\hline $\mathrm{In} 2^{\mathrm{i}}-\mathrm{Ba} 2-\mathrm{Ba} 5$ & $93.275(18)$ \\
\hline 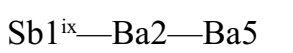 & $54.396(13)$ \\
\hline $\mathrm{Sb} 1-\mathrm{Ba} 2-\mathrm{Ba} 5$ & $54.396(13)$ \\
\hline $\mathrm{Sb} 3{ }^{\mathrm{i}}-\mathrm{Ba} 2-\mathrm{Ba} 5$ & $96.619(17)$ \\
\hline $\mathrm{Sb} 3-\mathrm{Ba} 2-\mathrm{Ba} 5$ & $96.619(17)$ \\
\hline $\mathrm{Sb} 22^{\mathrm{ii}}-\mathrm{Ba} 2-\mathrm{Ba} 5$ & $131.656(13)$ \\
\hline $\mathrm{Sb} 2^{\mathrm{i}}-\mathrm{Ba} 2-\mathrm{Ba} 5$ & $131.656(13)$ \\
\hline In $1^{\mathrm{vi}}-\mathrm{Ba} 2-\mathrm{Ba} 2^{\mathrm{i}}$ & $98.579(14)$ \\
\hline 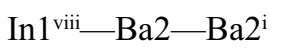 & $98.579(14)$ \\
\hline $\mathrm{In} 2^{\mathrm{ii}}-\mathrm{Ba} 2-\mathrm{Ba} 2^{\mathrm{i}}$ & $144.428(13)$ \\
\hline $\operatorname{In} 2^{\mathrm{i}}-\mathrm{Ba} 2-\mathrm{Ba} 2^{\mathrm{i}}$ & $144.428(13)$ \\
\hline $\mathrm{Sb} 1^{\mathrm{ix}}-\mathrm{Ba} 2-\mathrm{Ba} 2^{\mathrm{i}}$ & $95.073(13)$ \\
\hline $\mathrm{Sb} 1-\mathrm{Ba} 2-\mathrm{Ba} 2^{\mathrm{i}}$ & $95.073(13)$ \\
\hline $\mathrm{Sb} 3{ }^{\mathrm{i}}-\mathrm{Ba} 2-\mathrm{Ba}^{2}{ }^{\mathrm{i}}$ & $53.198(11)$ \\
\hline $\mathrm{Sb} 3-\mathrm{Ba} 2-\mathrm{Ba}^{\mathrm{i}}$ & $53.198(11)$ \\
\hline $\mathrm{Sb} 2^{\mathrm{ii}}-\mathrm{Ba} 2-\mathrm{Ba}^{2}{ }^{\mathrm{i}}$ & $133.895(11)$ \\
\hline $\mathrm{Sb} 2^{\mathrm{i}}-\mathrm{Ba} 2-\mathrm{Ba} 2^{\mathrm{i}}$ & $133.895(11)$ \\
\hline $\mathrm{Ba} 5-\mathrm{Ba} 2-\mathrm{Ba}^{\mathrm{i}}$ & $59.775(11)$ \\
\hline $\mathrm{Sb} 2^{\mathrm{x}}-\mathrm{Ba} 3-\mathrm{Sb} 2^{\mathrm{xi}}$ & $84.66(2)$ \\
\hline $\mathrm{Sb} 2^{x}-\mathrm{Ba} 3-\mathrm{Sb} 3^{\mathrm{xii}}$ & $97.024(18)$ \\
\hline $\mathrm{Sb} 2^{\mathrm{xi}}-\mathrm{Ba} 3-\mathrm{Sb} 3^{\mathrm{xii}}$ & $97.024(18)$ \\
\hline $\mathrm{Sb} 2^{\mathrm{x}}-\mathrm{Ba} 3-\mathrm{Sb} 4$ & $86.702(17)$ \\
\hline $\mathrm{Sb} 2^{\mathrm{xi}}-\mathrm{Ba} 3-\mathrm{Sb} 4$ & $86.702(17)$ \\
\hline $\mathrm{Sb} 3^{\mathrm{xii}}-\mathrm{Ba} 3-\mathrm{Sb} 4$ & $174.94(2)$ \\
\hline $\mathrm{Sb} 2^{\mathrm{x}}-\mathrm{Ba} 3-\mathrm{Sb} 1^{\mathrm{ix}}$ & $178.605(18)$ \\
\hline $\mathrm{Sb} 2^{\mathrm{xi}}-\mathrm{Ba} 3-\mathrm{Sb} 1^{\mathrm{ix}}$ & $94.053(13)$ \\
\hline $\mathrm{Sb} 3^{\mathrm{xii}}-\mathrm{Ba} 3-\mathrm{Sb} 1^{\mathrm{ix}}$ & $82.585(17)$ \\
\hline $\mathrm{Sb} 4-\mathrm{Ba} 3-\mathrm{Sb} 1^{\mathrm{ix}}$ & $93.769(17)$ \\
\hline $\mathrm{Sb} 2^{\mathrm{x}}-\mathrm{Ba} 3-\mathrm{Sb} 1^{\mathrm{i}}$ & $94.053(13)$ \\
\hline $\mathrm{Sb} 2^{\mathrm{xi}}-\mathrm{Ba} 3-\mathrm{Sb} 1^{\mathrm{i}}$ & $178.605(18)$ \\
\hline
\end{tabular}

\begin{tabular}{|c|c|}
\hline $\operatorname{In} 1^{\mathrm{xxiii}}-\operatorname{In} 1-\mathrm{Ba} 1^{\mathrm{i}}$ & $141.952(10)$ \\
\hline $\mathrm{Sb} 3^{\mathrm{xxiv}}-\mathrm{In} 1-\mathrm{Ba} 1^{\mathrm{i}}$ & $112.95(2)$ \\
\hline $\mathrm{Ba} 2^{\mathrm{xxiv}}-\mathrm{In} 1-\mathrm{Ba} 1^{\mathrm{i}}$ & $132.730(18)$ \\
\hline $\mathrm{Ba} 5^{\mathrm{xvi}}-\mathrm{In} 1-\mathrm{Ba} 1^{\mathrm{i}}$ & $129.15(2)$ \\
\hline $\mathrm{Sb} 4^{\mathrm{xxy}}-\operatorname{In} 2-\operatorname{In} 2^{\mathrm{ix}}$ & $111.322(17)$ \\
\hline $\mathrm{Sb} 4^{\mathrm{xxv}}-\mathrm{In} 2-\mathrm{Sb} 1^{\mathrm{ii}}$ & $109.93(2)$ \\
\hline $\operatorname{In} 2^{\mathrm{ix}}-\operatorname{In} 2-\mathrm{Sb} 1^{\mathrm{ii}}$ & $110.086(14)$ \\
\hline $\mathrm{Sb} 4^{\mathrm{xxy}}-\mathrm{In} 2-\mathrm{Sb} 2$ & $113.15(2)$ \\
\hline $\operatorname{In} 2^{\mathrm{ix}}-\operatorname{In} 2-\mathrm{Sb} 2$ & $108.733(14)$ \\
\hline 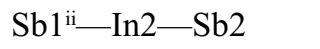 & $103.32(2)$ \\
\hline $\mathrm{Sb} 4^{\mathrm{xxv}}-\mathrm{In} 2-\mathrm{Ba} 2^{\mathrm{i}}$ & $173.81(3)$ \\
\hline $\operatorname{In} 2^{\mathrm{ix}}-\operatorname{In} 2-\mathrm{Ba} 2^{\mathrm{i}}$ & $65.488(10)$ \\
\hline 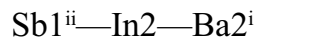 & $67.497(17)$ \\
\hline $\mathrm{Sb} 2-\mathrm{In} 2-\mathrm{Ba} 2^{\mathrm{i}}$ & $73.039(18)$ \\
\hline $\mathrm{Sb} 4^{\mathrm{xxv}}-\mathrm{In} 2-\mathrm{Ba}^{\mathrm{xxv}}$ & $58.068(19)$ \\
\hline $\operatorname{In} 2^{\mathrm{ix}}-\operatorname{In} 2-\mathrm{Ba}^{\mathrm{xxv}}$ & $140.012(10)$ \\
\hline $\mathrm{Sb} 1^{\mathrm{ii}}-\mathrm{In} 2-\mathrm{Ba} 3^{\mathrm{xxv}}$ & $109.592(19)$ \\
\hline $\mathrm{Sb} 2-\mathrm{In} 2-\mathrm{Ba}^{\mathrm{xxv}}$ & $56.626(15)$ \\
\hline $\mathrm{Ba} 2^{\mathrm{i}}-\mathrm{In} 2-\mathrm{Ba} 3^{\mathrm{xxv}}$ & $127.911(18)$ \\
\hline $\operatorname{In} 1^{\mathrm{xx}}-\mathrm{Sb} 1-\operatorname{In} 2^{\mathrm{ii}}$ & $101.88(2)$ \\
\hline $\mathrm{In} 1^{\mathrm{xx}}-\mathrm{Sb} 1-\mathrm{Ba} 1$ & $161.99(2)$ \\
\hline $\mathrm{In} 2^{\mathrm{ii}}-\mathrm{Sb} 1-\mathrm{Ba} 1$ & $85.363(18)$ \\
\hline $\operatorname{In} 1^{\mathrm{xx}}-\mathrm{Sb} 1-\mathrm{Ba} 1^{\mathrm{iii}}$ & $75.959(17)$ \\
\hline $\operatorname{In} 2^{\mathrm{ii}}-\mathrm{Sb} 1-\mathrm{Ba} 1^{\mathrm{iii}}$ & $79.632(17)$ \\
\hline $\mathrm{Ba} 1-\mathrm{Sb} 1-\mathrm{Ba} 1^{\mathrm{iii}}$ & $89.375(16)$ \\
\hline $\mathrm{In} 1^{\mathrm{xx}}-\mathrm{Sb} 1-\mathrm{Ba} 3^{\mathrm{i}}$ & 84.155 (19) \\
\hline $\operatorname{In} 2^{\mathrm{ii}}-\mathrm{Sb} 1-\mathrm{Ba} 3^{\mathrm{i}}$ & $161.77(2)$ \\
\hline $\mathrm{Ba} 1-\mathrm{Sb} 1-\mathrm{Ba} 3^{\mathrm{i}}$ & $84.272(16)$ \\
\hline $\mathrm{Ba} 1^{\mathrm{iii}}-\mathrm{Sb} 1-\mathrm{Ba}^{\mathrm{i}}$ & $85.293(16)$ \\
\hline $\mathrm{In} 1^{\mathrm{xx}}-\mathrm{Sb} 1-\mathrm{Ba} 2$ & $117.27(2)$ \\
\hline $\mathrm{In} 2^{\mathrm{ii}}-\mathrm{Sb} 1-\mathrm{Ba} 2$ & $63.114(17)$ \\
\hline $\mathrm{Ba} 1-\mathrm{Sb} 1-\mathrm{Ba} 2$ & $80.729(16)$ \\
\hline $\mathrm{Ba} 1^{\mathrm{iii}}-\mathrm{Sb} 1-\mathrm{Ba} 2$ & $141.977(19)$ \\
\hline $\mathrm{Ba} 3^{\mathrm{i}}-\mathrm{Sb} 1-\mathrm{Ba} 2$ & $129.426(19)$ \\
\hline $\mathrm{In} 1^{\mathrm{xx}}-\mathrm{Sb} 1-\mathrm{Ba} 5$ & $62.606(19)$ \\
\hline $\mathrm{In} 2^{\mathrm{ii}}-\mathrm{Sb} 1-\mathrm{Ba} 5$ & $117.648(19)$ \\
\hline $\mathrm{Ba} 1-\mathrm{Sb} 1-\mathrm{Ba} 5$ & $128.543(19)$ \\
\hline $\mathrm{Ba} 1^{\mathrm{iii}}-\mathrm{Sb} 1-\mathrm{Ba} 5$ & $137.18(2)$ \\
\hline $\mathrm{Ba} 3^{\mathrm{i}}-\mathrm{Sb} 1-\mathrm{Ba} 5$ & $80.461(15)$ \\
\hline $\mathrm{Ba} 2-\mathrm{Sb} 1-\mathrm{Ba} 5$ & $72.411(18)$ \\
\hline $\operatorname{In} 1^{\mathrm{xxvi}}-\mathrm{Sb} 2-\operatorname{In} 2$ & $118.55(2)$ \\
\hline $\mathrm{In} 1^{\mathrm{xxv}}-\mathrm{Sb} 2-\mathrm{Ba}^{\mathrm{xxv}}$ & $158.88(2)$ \\
\hline $\mathrm{In} 2-\mathrm{Sb} 2-\mathrm{Ba}^{\mathrm{xxv}}$ & $78.130(18)$ \\
\hline $\mathrm{In} 1^{\mathrm{xxvi}}-\mathrm{Sb} 2-\mathrm{Ba} 1^{\mathrm{ii}}$ & $82.178(18)$ \\
\hline $\mathrm{In} 2-\mathrm{Sb} 2-\mathrm{Ba} 1^{\mathrm{ii}}$ & $84.879(18)$ \\
\hline $\mathrm{Ba} 3^{\mathrm{xxv}}-\mathrm{Sb} 2-\mathrm{Ba}^{\mathrm{ii}}$ & $86.909(16)$ \\
\hline In $1^{x x v i}-\mathrm{Sb} 2-B a 1^{\text {xxvii }}$ & $75.772(18)$ \\
\hline $\mathrm{In} 2-\mathrm{Sb} 2-\mathrm{Ba} 1^{\mathrm{xxvii}}$ & $159.71(2)$ \\
\hline
\end{tabular}




\begin{tabular}{|c|c|}
\hline $\mathrm{Sb} 3^{\mathrm{xii}}-\mathrm{Ba} 3-\mathrm{Sb} 1^{\mathrm{i}}$ & $82.585(17)$ \\
\hline $\mathrm{Sb} 4-\mathrm{Ba} 3-\mathrm{Sb}^{\mathrm{i}}$ & $93.769(17)$ \\
\hline $\mathrm{Sb} 1^{\mathrm{ix}}-\mathrm{Ba} 3-\mathrm{Sb} 1^{\mathrm{i}}$ & $87.23(2)$ \\
\hline $\mathrm{Sb} 2^{\mathrm{x}}-\mathrm{Ba} 3-\operatorname{In} 2^{\mathrm{x}}$ & $45.244(13)$ \\
\hline 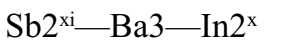 & $91.297(18)$ \\
\hline $\mathrm{Sb} 3^{\mathrm{xii}}-\mathrm{Ba} 3-\operatorname{In} 2^{\mathrm{x}}$ & $140.454(14)$ \\
\hline $\mathrm{Sb} 4-\mathrm{Ba} 3-\operatorname{In} 2^{\mathrm{x}}$ & $42.412(12)$ \\
\hline $\mathrm{Sb} 1^{\mathrm{ix}}-\mathrm{Ba} 3-\operatorname{In} 2^{\mathrm{x}}$ & $135.44(2)$ \\
\hline 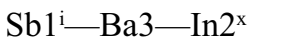 & $88.172(14)$ \\
\hline $\mathrm{Sb} 2^{\mathrm{x}}-\mathrm{Ba} 3-\operatorname{In} 2^{\mathrm{xi}}$ & $91.297(18)$ \\
\hline $\mathrm{Sb} 2^{\mathrm{xi}}-\mathrm{Ba} 3-\operatorname{In} 2^{\mathrm{xi}}$ & $45.244(13)$ \\
\hline $\mathrm{Sb} 3^{\mathrm{xii}}-\mathrm{Ba} 3-\mathrm{In} 2^{\mathrm{xi}}$ & $140.454(14)$ \\
\hline $\mathrm{Sb} 4-\mathrm{Ba} 3-\mathrm{In} 2^{\mathrm{xi}}$ & $42.412(12)$ \\
\hline $\mathrm{Sb} 1^{\mathrm{ix}}-\mathrm{Ba} 3-\operatorname{In} 2^{\mathrm{xi}}$ & $88.172(14)$ \\
\hline $\mathrm{Sb} 1^{\mathrm{i}}-\mathrm{Ba} 3-\mathrm{In} 2^{\mathrm{xi}}$ & $135.44(2)$ \\
\hline $\operatorname{In} 2^{x}-B a 3-\operatorname{In} 2^{x i}$ & $65.310(18)$ \\
\hline $\mathrm{Sb} 2^{x}-\mathrm{Ba} 3-\mathrm{Ba} 5$ & $130.270(14)$ \\
\hline $\mathrm{Sb} 2^{\mathrm{xi}}-\mathrm{Ba} 3-\mathrm{Ba} 5$ & $130.270(14)$ \\
\hline $\mathrm{Sb} 3^{\mathrm{xii}}-\mathrm{Ba} 3-\mathrm{Ba} 5$ & $51.447(18)$ \\
\hline $\mathrm{Sb} 4-\mathrm{Ba} 3-\mathrm{Ba} 5$ & $123.49(2)$ \\
\hline $\mathrm{Sb} 1^{\mathrm{ix}}-\mathrm{Ba} 3-\mathrm{Ba} 5$ & $50.390(12)$ \\
\hline $\mathrm{Sb} 1-\mathrm{Ba} 3-\mathrm{Ba} 5$ & $50.390(12)$ \\
\hline $\operatorname{In} 2^{x}-\mathrm{Ba} 3-\mathrm{Ba} 5$ & $138.151(14)$ \\
\hline $\operatorname{In} 2^{\mathrm{xi}}-\mathrm{Ba} 3-\mathrm{Ba} 5$ & $138.151(14)$ \\
\hline $\mathrm{Sb} 2^{\mathrm{x}}-\mathrm{Ba} 3-\mathrm{Ba} 1^{\mathrm{ix}}$ & $132.982(18)$ \\
\hline $\mathrm{Sb} 2^{\mathrm{xi}}-\mathrm{Ba} 3-\mathrm{Ba}^{\mathrm{ix}}$ & $91.140(13)$ \\
\hline $\mathrm{Sb} 3^{\mathrm{xii}}-\mathrm{Ba} 3-\mathrm{Ba} 1^{\mathrm{ix}}$ & $129.919(16)$ \\
\hline $\mathrm{Sb} 4-\mathrm{Ba} 3-\mathrm{Ba}^{\mathrm{ix}}$ & $46.281(12)$ \\
\hline $\mathrm{Sb} 1^{\mathrm{ix}}-\mathrm{Ba} 3-\mathrm{Ba} 1^{\mathrm{ix}}$ & $47.495(11)$ \\
\hline $\mathrm{Sb} 1^{\mathrm{i}}-\mathrm{Ba} 3-\mathrm{Ba}^{\mathrm{ix}}$ & $90.133(16)$ \\
\hline $\operatorname{In} 2^{x}-B a 3-B a 1^{\text {ix }}$ & $88.244(15)$ \\
\hline 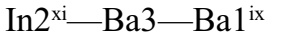 & $55.877(11)$ \\
\hline $\mathrm{Ba} 5-\mathrm{Ba} 3-\mathrm{Ba} 1^{\mathrm{ix}}$ & $86.529(14)$ \\
\hline $\mathrm{Sb} 2^{\mathrm{x}}-\mathrm{Ba} 3-\mathrm{Ba} 1^{\mathrm{i}}$ & $91.140(13)$ \\
\hline $\mathrm{Sb} 2^{\mathrm{xi}}-\mathrm{Ba} 3-\mathrm{Ba} 1^{\mathrm{i}}$ & $132.982(18)$ \\
\hline $\mathrm{Sb} 3^{\mathrm{xii}}-\mathrm{Ba} 3-\mathrm{Ba}^{\mathrm{i}}$ & $129.919(16)$ \\
\hline $\mathrm{Sb} 4-\mathrm{Ba} 3-\mathrm{Ba} 1^{\mathrm{i}}$ & $46.281(12)$ \\
\hline 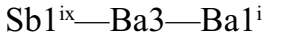 & $90.133(16)$ \\
\hline 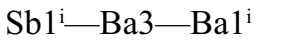 & $47.495(11)$ \\
\hline $\operatorname{In} 2^{x}-B a 3-B a 1^{i}$ & $55.877(11)$ \\
\hline $\operatorname{In} 2^{\mathrm{xi}}-\mathrm{Ba} 3-\mathrm{Ba} 1^{\mathrm{i}}$ & $88.244(15)$ \\
\hline $\mathrm{Ba} 5-\mathrm{Ba} 3-\mathrm{Ba}^{\mathrm{i}}$ & $86.529(14)$ \\
\hline $\mathrm{Ba} 1^{\mathrm{ix}}-\mathrm{Ba} 3-\mathrm{Ba} 1^{\mathrm{i}}$ & $58.870(14)$ \\
\hline $\mathrm{Sb} 2^{\mathrm{x}}-\mathrm{Ba} 3-\mathrm{Ba} 1^{\mathrm{xii}}$ & $48.100(12)$ \\
\hline $\mathrm{Sb} 2^{\mathrm{xi}}-\mathrm{Ba} 3-\mathrm{Ba} 1^{\mathrm{xii}}$ & $89.545(17)$ \\
\hline $\mathrm{Sb} 3^{\mathrm{xii}}-\mathrm{Ba} 3-\mathrm{Ba} 1^{\mathrm{xii}}$ & $48.968(13)$ \\
\hline $\mathrm{Sb} 4-\mathrm{Ba} 3-\mathrm{Ba}^{\mathrm{xii}}$ & $134.789(16)$ \\
\hline $\mathrm{Sb} 1^{\mathrm{ix}}-\mathrm{Ba} 3-\mathrm{Ba} 1^{\mathrm{xii}}$ & $131.442(18)$ \\
\hline
\end{tabular}

\begin{tabular}{|c|c|}
\hline $\mathrm{Ba} 3^{\mathrm{xxy}}-\mathrm{Sb} 2-\mathrm{Ba} 1^{\mathrm{xxvii}}$ & 85.014 (17) \\
\hline $\mathrm{Ba} 1^{\mathrm{ii}}-\mathrm{Sb} 2-\mathrm{Ba} 1^{\mathrm{xxvii}}$ & $82.998(15)$ \\
\hline $\mathrm{In} 1^{\mathrm{xxvi}}-\mathrm{Sb} 2-\mathrm{Ba} 4^{\mathrm{xiv}}$ & $93.71(2)$ \\
\hline $\mathrm{In} 2-\mathrm{Sb} 2-\mathrm{Ba} 4^{\mathrm{xiv}}$ & $96.72(2)$ \\
\hline $\mathrm{Ba}^{3 x y}-\mathrm{Sb} 2-\mathrm{Ba} 4^{\mathrm{xiv}}$ & $97.149(16)$ \\
\hline $\mathrm{Ba} 1^{\mathrm{ii}}-\mathrm{Sb} 2-\mathrm{Ba} 4^{\mathrm{xiv}}$ & $175.863(18)$ \\
\hline $\mathrm{Ba} 1^{\mathrm{xxvii}}-\mathrm{Sb} 2-\mathrm{Ba} 4^{\mathrm{xiv}}$ & 96.509 (19) \\
\hline $\operatorname{In} 1^{\mathrm{xxvi}}-\mathrm{Sb} 2-\mathrm{Ba} 2^{\mathrm{i}}$ & $59.314(17)$ \\
\hline $\mathrm{In} 2-\mathrm{Sb} 2-\mathrm{Ba} 2^{\mathrm{i}}$ & $59.238(16)$ \\
\hline $\mathrm{Ba}^{\mathrm{xxv}}-\mathrm{Sb} 2-\mathrm{Ba}^{\mathrm{i}}$ & $135.28(2)$ \\
\hline $\mathrm{Ba} 1^{\mathrm{ii}}-\mathrm{Sb} 2-\mathrm{Ba} 2^{\mathrm{i}}$ & $77.125(15)$ \\
\hline $\mathrm{Ba} 1^{\mathrm{xxvii}}-\mathrm{Sb} 2-\mathrm{Ba} 2^{\mathrm{i}}$ & $132.629(18)$ \\
\hline $\mathrm{Ba} 4^{\mathrm{xiv}}-\mathrm{Sb} 2-\mathrm{Ba} 2^{\mathrm{i}}$ & $100.382(15)$ \\
\hline $\operatorname{In} 1^{\mathrm{vii}}-\mathrm{Sb} 3-\operatorname{In} 1^{\mathrm{xxvi}}$ & $119.85(3)$ \\
\hline $\mathrm{In} 1^{\mathrm{vii}}-\mathrm{Sb} 3-\mathrm{Ba} 3^{\mathrm{vi}}$ & $81.640(18)$ \\
\hline In $1^{\mathrm{xxvi}}-\mathrm{Sb} 3-\mathrm{Ba} 3^{\mathrm{vi}}$ & $81.641(18)$ \\
\hline In $1^{\mathrm{vii}}-\mathrm{Sb} 3-\mathrm{Ba} 2^{\mathrm{i}}$ & $123.96(2)$ \\
\hline $\mathrm{In} 1^{\mathrm{xxvi}}-\mathrm{Sb} 3-\mathrm{Ba} 2^{\mathrm{i}}$ & $61.434(16)$ \\
\hline $\mathrm{Ba} 3^{\mathrm{vi}}-\mathrm{Sb} 3-\mathrm{Ba}^{\mathrm{i}}$ & $141.785(14)$ \\
\hline In $1^{\text {vii }-S b 3-B a 2 ~}$ & $61.434(16)$ \\
\hline In $1^{x x v i}-\mathrm{Sb} 3-\mathrm{Ba} 2$ & $123.96(2)$ \\
\hline $\mathrm{Ba} 3^{\mathrm{vi}}-\mathrm{Sb} 3-\mathrm{Ba} 2$ & $141.785(14)$ \\
\hline $\mathrm{Ba} 2{ }^{\mathrm{i}}-\mathrm{Sb} 3-\mathrm{Ba} 2$ & $73.60(2)$ \\
\hline In $1^{\mathrm{vii}}-\mathrm{Sb} 3-\mathrm{Ba} 1$ & $77.750(14)$ \\
\hline In $1^{\text {xxvi }-S b 3-B a 1}$ & $154.85(2)$ \\
\hline $\mathrm{Ba}^{\mathrm{vi}}{ }^{\mathrm{vi}} \mathrm{Sb} 3-\mathrm{Ba} 1$ & 83.695 (17) \\
\hline $\mathrm{Ba} 2{ }^{\mathrm{i}}-\mathrm{Sb} 3-\mathrm{Ba} 1$ & $126.13(2)$ \\
\hline $\mathrm{Ba} 2-\mathrm{Sb} 3-\mathrm{Ba} 1$ & 79.407 (14) \\
\hline In $1^{\mathrm{vii}}-\mathrm{Sb} 3-\mathrm{Ba} 1^{\mathrm{ii}}$ & $154.85(2)$ \\
\hline In $1^{\mathrm{xxvi}}-\mathrm{Sb} 3-\mathrm{Ba} 1^{\mathrm{ii}}$ & $77.750(14)$ \\
\hline $\mathrm{Ba} 3^{\mathrm{vi}}-\mathrm{Sb} 3-\mathrm{Ba} 1^{\mathrm{ii}}$ & 83.695 (17) \\
\hline $\mathrm{Ba} 2^{\mathrm{i}}-\mathrm{Sb} 3-\mathrm{Ba} 1^{\mathrm{ii}}$ & 79.407 (14) \\
\hline $\mathrm{Ba} 2-\mathrm{Sb} 3-\mathrm{Ba}^{\mathrm{ii}}$ & $126.13(2)$ \\
\hline $\mathrm{Ba} 1-\mathrm{Sb} 3-\mathrm{Ba}^{\mathrm{ii}}$ & $80.42(2)$ \\
\hline In $1^{\mathrm{vii}}-\mathrm{Sb} 3-\mathrm{Ba} 5^{\mathrm{xxviii}}$ & $60.170(15)$ \\
\hline 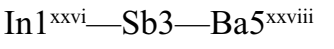 & $60.170(15)$ \\
\hline $\mathrm{Ba}^{\mathrm{vi}}-\mathrm{Sb} 3-\mathrm{Ba}^{\mathrm{xxviii}}$ & $80.13(2)$ \\
\hline $\mathrm{Ba} 2^{\mathrm{i}}-\mathrm{Sb} 3-\mathrm{Ba} 5^{\mathrm{xxviii}}$ & 89.005 (18) \\
\hline $\mathrm{Ba} 2-\mathrm{Sb} 3-\mathrm{Ba} 5^{\mathrm{xxviii}}$ & $89.005(18)$ \\
\hline $\mathrm{Ba} 1-\mathrm{Sb} 3-\mathrm{Ba}^{\mathrm{xxviii}}$ & $136.530(13)$ \\
\hline $\mathrm{Ba} 1^{\mathrm{ii}}-\mathrm{Sb} 3-\mathrm{Ba} 5^{\mathrm{xxviii}}$ & $136.530(13)$ \\
\hline $\operatorname{In} 2^{x}-\operatorname{Sb} 4-\operatorname{In} 2^{x i}$ & $103.75(3)$ \\
\hline $\operatorname{In} 2^{x}-\operatorname{Sb} 4-B a 1^{\text {ix }}$ & $162.15(3)$ \\
\hline $\operatorname{In} 2^{\mathrm{xi}}-\mathrm{Sb} 4-\mathrm{Ba} 1^{\mathrm{ix}}$ & $83.388(15)$ \\
\hline $\operatorname{In} 2^{x}-\mathrm{Sb} 4-\mathrm{Ba}^{\mathrm{i}}$ & $83.388(15)$ \\
\hline $\operatorname{In} 2^{\mathrm{xi}}-\mathrm{Sb} 4-\mathrm{Ba} 1^{\mathrm{i}}$ & $162.15(3)$ \\
\hline $\mathrm{Ba} 1^{\mathrm{ix}}-\mathrm{Sb} 4-\mathrm{Ba} 1^{\mathrm{i}}$ & $85.40(2)$ \\
\hline $\mathrm{In} 2^{\mathrm{x}}-\mathrm{Sb} 4-\mathrm{Ba} 3$ & $79.52(2)$ \\
\hline
\end{tabular}


supporting information

\begin{tabular}{|c|c|c|c|}
\hline $\mathrm{Sb} 1^{\mathrm{i}}-\mathrm{Ba} 3-\mathrm{Ba} 1^{\mathrm{xii}}$ & $89.193(13)$ & $\mathrm{In} 2^{\mathrm{xi}}-\mathrm{Sb} 4-\mathrm{Ba} 3$ & $79.52(2)$ \\
\hline $\operatorname{In} 2^{x}-\mathrm{Ba} 3-\mathrm{Ba}^{\mathrm{xii}}$ & $92.751(13)$ & $\mathrm{Ba} 1^{\mathrm{ix}}-\mathrm{Sb} 4-\mathrm{Ba} 3$ & 85.867 (17) \\
\hline In $2^{\mathrm{xi}}-\mathrm{Ba} 3-\mathrm{Ba} 1^{\mathrm{xii}}$ & $125.221(17)$ & $\mathrm{Ba} 1^{\mathrm{i}}-\mathrm{Sb} 4-\mathrm{Ba} 3$ & 85.867 (17) \\
\hline $\mathrm{Ba} 5-\mathrm{Ba} 3-\mathrm{Ba} 1^{\mathrm{xii}}$ & $92.258(15)$ & $\mathrm{In} 2^{\mathrm{x}}-\mathrm{Sb} 4-\mathrm{Ba} 4$ & $97.21(2)$ \\
\hline $\mathrm{Ba} 1^{\mathrm{ix}}-\mathrm{Ba} 3-\mathrm{Ba} 1^{\mathrm{xii}}$ & $178.779(18)$ & $\mathrm{In} 2^{\mathrm{xi}}-\mathrm{Sb} 4-\mathrm{Ba} 4$ & $97.21(2)$ \\
\hline $\mathrm{Ba} 1^{\mathrm{i}}-\mathrm{Ba} 3-\mathrm{Ba} 1^{\mathrm{xii}}$ & $121.212(11)$ & $\mathrm{Ba} 1^{\mathrm{ix}}-\mathrm{Sb} 4-\mathrm{Ba} 4$ & $98.083(19)$ \\
\hline $\mathrm{Sb} 2^{\mathrm{xiii}}-\mathrm{Ba} 4-\mathrm{Sb} 2^{\mathrm{xiv}}$ & $135.28(3)$ & 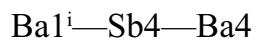 & $98.083(19)$ \\
\hline $\mathrm{Sb} 2^{\mathrm{xiii}}-\mathrm{Ba} 4-\mathrm{Sb} 2^{\text {viii }}$ & $82.364(19)$ & $\mathrm{Ba} 3-\mathrm{Sb} 4-\mathrm{Ba} 4$ & $174.60(3)$ \\
\hline
\end{tabular}

Symmetry codes: (i) $-x+1 / 2,-y+1 / 2, z$; (ii) $-x+1 / 2, y, z$; (iii) $-x,-y,-z+1$; (iv) $x-1 / 2,-y,-z$; (v) $-x,-y,-z$; (vi) $-x+1 / 2,-y+1 / 2, z-1$; (vii) $x-1 / 2, y-1 / 2$, $-z$; (viii) $x-1 / 2,-y+1,-z$; (ix) $x,-y+1 / 2, z$; (x) $-x+1, y+1 / 2,-z+1$; (xi) $x-1 / 2, y+1 / 2,-z+1$; (xii) $-x+1 / 2,-y+1 / 2, z+1$; (xiii) $x-1 / 2, y+1 / 2,-z$; (xiv) $-x+1$, $-y+1,-z$; (xv) $-x+1, y+1 / 2,-z$; (xvi) $-x+1 / 2,-y+3 / 2, z$; (xvii) $-x+1,-y+1,-z+1$; (xviii) $x-1 / 2,-y+1,-z+1$; (xix) $-x+1, y-1 / 2,-z+1$; (xx) $x-1 / 2, y-1 / 2$, $-z+1$; (xxi) $x, y, z+1$; (xxii) $x+1 / 2, y+1 / 2,-z+1$; (xxiii) $x,-y+3 / 2, z$; (xxiv) $x+1 / 2, y+1 / 2,-z$; (xxv) $x+1 / 2, y-1 / 2,-z+1$; (xxvi) $-x+1, y-1 / 2,-z$; (xxvii) $x+1 / 2,-y,-z$; (xxviii) $x, y, z-1$. 